\title{
Rapid Body Scanning Technology for a Virtual Mass Customization Process in Garment Industry
}

\author{
Michael ERNST ${ }^{\star a, b}$, Monika BOEHM ${ }^{a}$, Ute DETERING-KOLL ${ }^{\star a}$, Deidre WINDVOGEL ${ }^{\mathrm{b}}$ \\ ${ }^{a}$ Niederrhein University of Applied Sciences, Germany; \\ ${ }^{\mathrm{b}}$ Cape Peninsula University of Technology, South Africa
}

DOI: $10.15221 / 16.159$ http://dx.doi.org/10.15221/16.159

\begin{abstract}
Nowadays the product development process in apparel industry is mainly influenced by the customercustomizing products become more and more popular aiming not only to improve fit but also to individualize and satisfy customer needs. Numerous tools have been developed all facing the same problems: How to take body measurements, how to take the right measurements, how to transport individual posture information [2, 3] and how to implement these data correctly into a product pattern based on a predefined construction system. Therefore body measurements and posture information both have to be linked by transfer equations to finish/ready measurements positioned on defined locations of the construction and finally of the product.

Most of the companies offering mass customization to their clients are working without scanning technology and without a fitting session- checking fit and design only in a very last process step- if at all-usually when the product is already manufactured

Virtual Product Development is a powerful tool- not only for merchandising and promotion but also for design development, fit check and technical product development, closing the gap between missing real products and missing imagination of customers.
\end{abstract}

Keywords: 3D full body scanning technology; body posture, body shape; sizing and fit, 3D simulation, virtual product development; mass customization

\section{Introduction}

Fit is the most critical part of a successful "made to measure" process in garment industry [1]. First of all individual realistic body measurements must be available representing exactly the customer.

Taking these measurements manually is leading to uncertain measurement locations, operator dependent tape measure handling, lost information and the fact, that the process is usually not repeatable. 3D full body scanning technology can close this gap by offering a "stored measurement situation" which can be analyzed and re-measured again and again on one side and by freezing much more valuable information related to body posture and shape on the other side [6].

Unfortunately the information generated with this technology is only good as the quality of the scan, meaning that the scanning process has to be prepared and monitored carefully concerning posture dependency of measurements [4].

The obtained data have to be transferred to the product pattern by using CAD technology- usually by using a so called $\mathrm{mtm}$ (made to measure) grading- a second grading overlaid. But regarding the mix of data obtained within the scanning process- especially body shape and posture information- and expecting a benefit by implementing this mix in a whole- new ideas and concepts are needed.

Within the mtm process also an early testing procedure is necessary to check the fit directly in advance before starting with the manufacturing process. Therefore 3D simulation technology seems to be the method of choice- testing the individualized pattern on a so called scanatar, generated directly from the scan [5].

In this paper the mentioned data mix for made to measure garments based on 3D full body scanning technology is discussed and awareness for the meaning of controlled scanning conditions and scanning information should be created.

*michael.ernst@hs-niederrhein.de ;+49 2161 186-6080;

*ute.detering-koll@hs-niederrhein.de; +49 2161 186-6081 


\section{3D full body scanning information}

The parameters for 3D body scanning are given by the scanning technology on one side and the scanning procedure on the other side. Apart from different scanning technologies, calibration aspects and flexibility in traveling the scanning subject itself is the most important part of the process.

Body scanning technology for apparel industry is strongly related to design aspects and linked directly to sizing and fit in a technical product development process.

For all these scanning processes the moment of scanning freezes a 3D picture of a person and represents only a snap-shot- more or less reflecting the reality. Regarding bodies of human beings in detail show motion and thereby change all the time. The parameters are therefore given by the subject itself and have to be controlled compulsory. Because of the individual bodies and individual behavior chest motion during breathing, posture and position is also an individual matter but possible to control instead of physical and mental aspects- e.g. personal well-being of the day.

Although the process of generating scanatars can become tricky for special target groups, e.g. plus size female- and the scanning position has to be changed to get proper data, these scanatars are equivalent to material characteristics and the pattern itself in a $3 \mathrm{D}$ simulation and contribute significantly to its success [3].

The following information can be extracted from 3D full body scans for mtm garments:

Body measurements (according to construction systems, standards; automatically programmed)

Additional measurements taken directly from the scan if needed by using the measurement software (re-measure, product dependent, control function)

Posture information (hollow back, shape of legs, hip position,...)

Body contour information (hip curve; side seam, center back, buttocks, bust shape,, ..)

This mix of multiple information offers a detailed picture of the customer and enables the user to create $\mathrm{mtm}$ pattern with a proper fit.

The measurements can be transferred directly to individual body measurement charts of macro tools or assigned in a second grading to the pattern using the mtm tools of CAD suppliers.

The posture information can be impelemented into the CAD pattern by modelling the basic blocks. The body contour information can be used for modelling the pattern lines by sloping or opening locally the contour and matching them directly to the $2 \mathrm{D}$ information given by the scan.

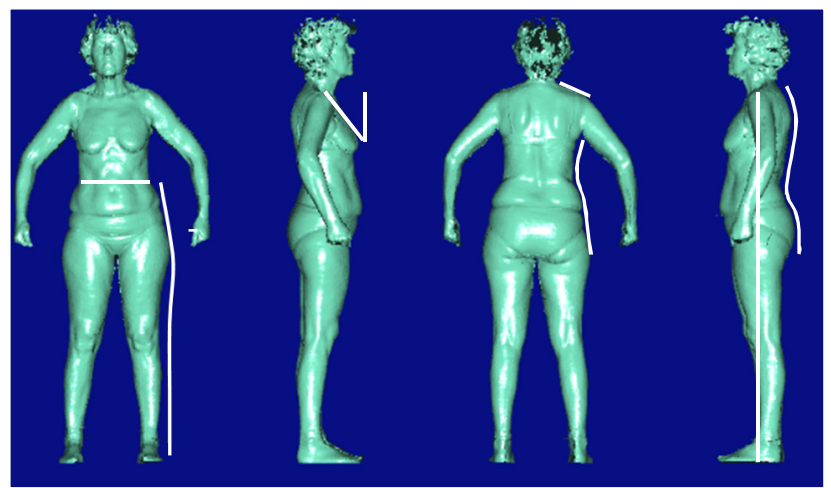

Fig. 1: 3D full body scanning information- example of measurements- shape- contour information

\section{Research methodology}

Investigations have been carried out using a sample size of more than 30 different female $\left(\mathrm{F}^{\mathrm{n}}\right)$ and male $(\mathrm{Mn})$ bodies- covering the ageing structure from 18 to 59 years old.

All subjects have been scanned in a first step with different 3D full Body scanning systems- laser, depth bed sensor, white light- and in different postures following the research methodology shown in fig. 2. 


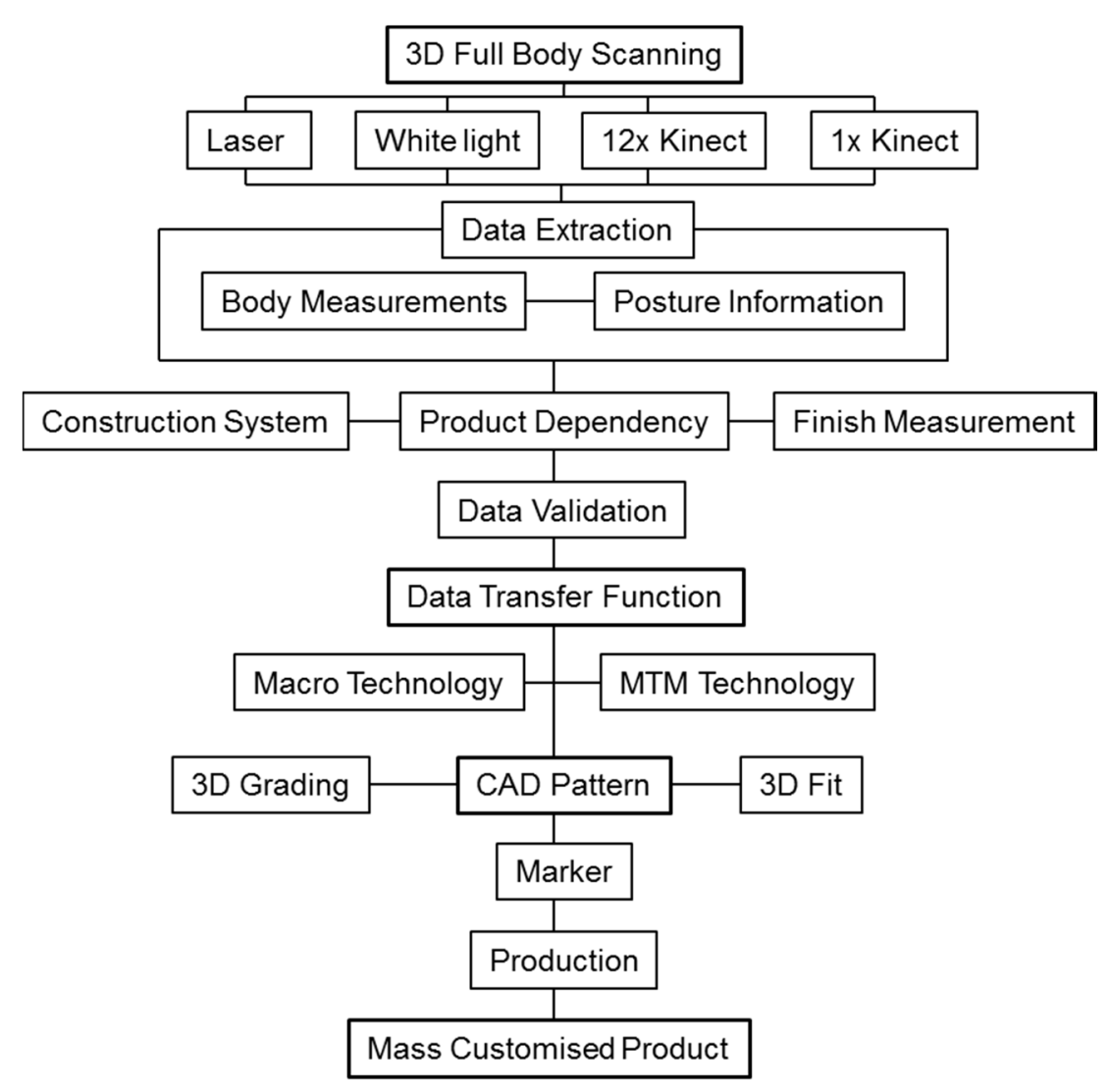

Fig. 2: research methodology - rapid body scanning for virtual mass customization

\subsection{Body scanning technology}

Scanning was realized using various 3D full body scanning technologies:

- Anthroscan; Human Solutions GmbH; Laser Scanning Technology (L); VirLab Niederrhein

- Ditus Smart; Human Solutions GmbH; Kinect Sensor Technology 12x Kinect (K12); VirLab Niederrhein

- StyKu; Styku; Kinect Sensor Technology 1x Kinect (K1), turntable; VirLab Nlederrhein

- $\quad \mathrm{TC}^{2}$; NX 12; white light projection (W); Technology Station TSCT, Belleville

And compared to manually $(\mathrm{M})$ taken measurements and photo-scanning technology $(\mathrm{P})$.

Posture and pose dependent influences:

- Standard scan position

- Individual relaxed position

- Heelless, 3-5-10 cm heel (female)

The resulting scans have been validated and tested against the localization of measurements needed for the follow up mass customized pattern making process. Before starting a manufacturing process the gained product development was checked by using $3 \mathrm{~d}$ virtual prototyping systems and implementing scanatars automatically adapted parametric mannequins or derived statistical avatars from sizing surveys.

In a first step the extracted data are necessarily validated for the localization of the measurements and the body posture and assigned to:

- parametric pattern prepared for a mass customization process (MTM CAD tools)

- macro pattern constructions

- given parametric mannequins (to avoid showing scanatars if not accepted) 


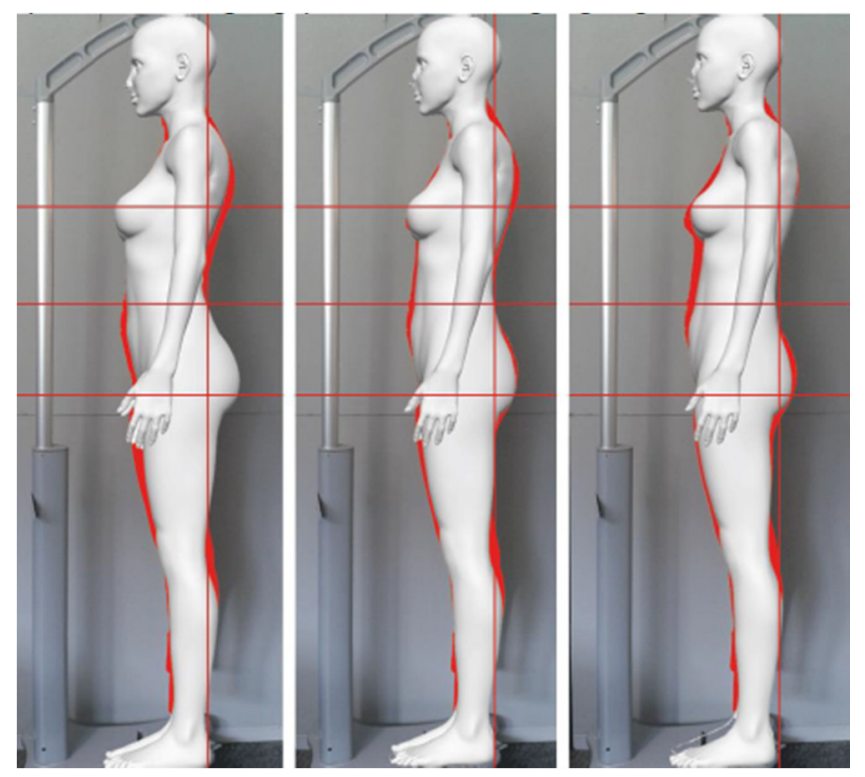

Fig. 3: Automatic measurement data transfer to parametric mannequin and posture variation- Size Germany N38 Reference- Grafis-Vstitcher parametric mannequin Deborah

Using the Humans Solutions scanning software enables to generate so called scanatars using a special wizard guiding through the process [3].The scanatar is an avatar from an individual person and represents the virtual mannequin of a real person with identical body measurements and shapes. Two scan positions (scan and relaxed position) are necessary- already included in the scanning test procedure in this project. The obj scanatar files from Styku are prepared for virtual fitting by opening crotch and armhole areas.

\subsection{CAD Pattern}

\subsubsection{Macro technology}

Product related body measurements, generated with body scanning technology and validated are directly implemented to macro tools of different CAD supplier: Grafis, Gerber wizard, smart developer/smart pattern assyst, Gemini CAD. The macros are built up with multiple variables representing directly body measurements, add on values or calculated measurements.

In fig 4 Gemini macro MtM tool with a construction grid and MTM server import for data transfer are shown.

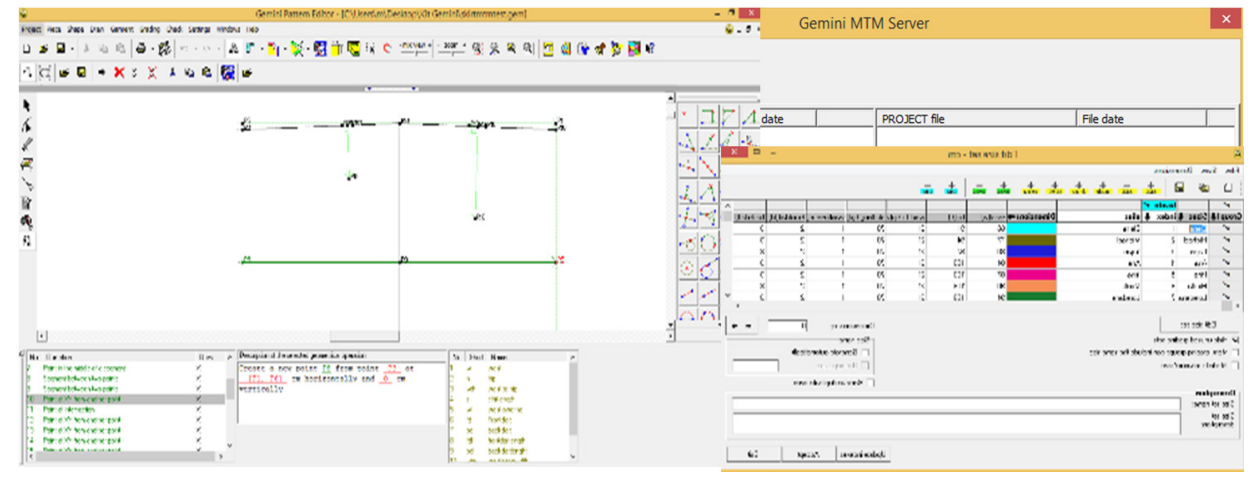

Fig. 4: MtM Macro and MtM Macro server of Gemini CAD 


\subsubsection{MtM Grading}

Product related body measurements are also transferred to so called $\mathrm{mtm}$ grading tools offered from various software suppliers: assyst $m t m$, Lectra $f t$ net,... and assigned directly as change values +/- to already set up standard gradings. In fig. 5 a men's trousers grading in Lectra modaris expert is shown.

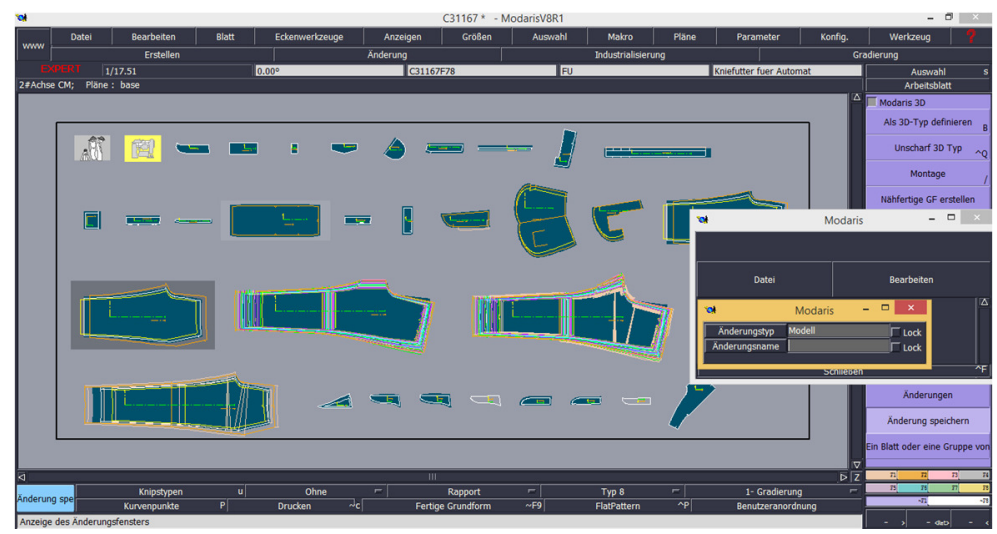

Fig. 5: mtm Fit Net Lectra Modaris expert

\subsubsection{D Grading}

A new tool offered from assyst is investigated taking grade rules directly from avatars in relation to statistical avatars from isize system. With reference to a best fit avatar the $\mathrm{mtm}$ grading can be calculated- with reference to body locations or directly product related by transferring the 3D lines of the product- simulated in vidya assyst- to the avatar. Thus it is possible to detect easily the right grading positions for tight fitted garments and assigning the values measured with 3D grading to the pattern in vidyacad.

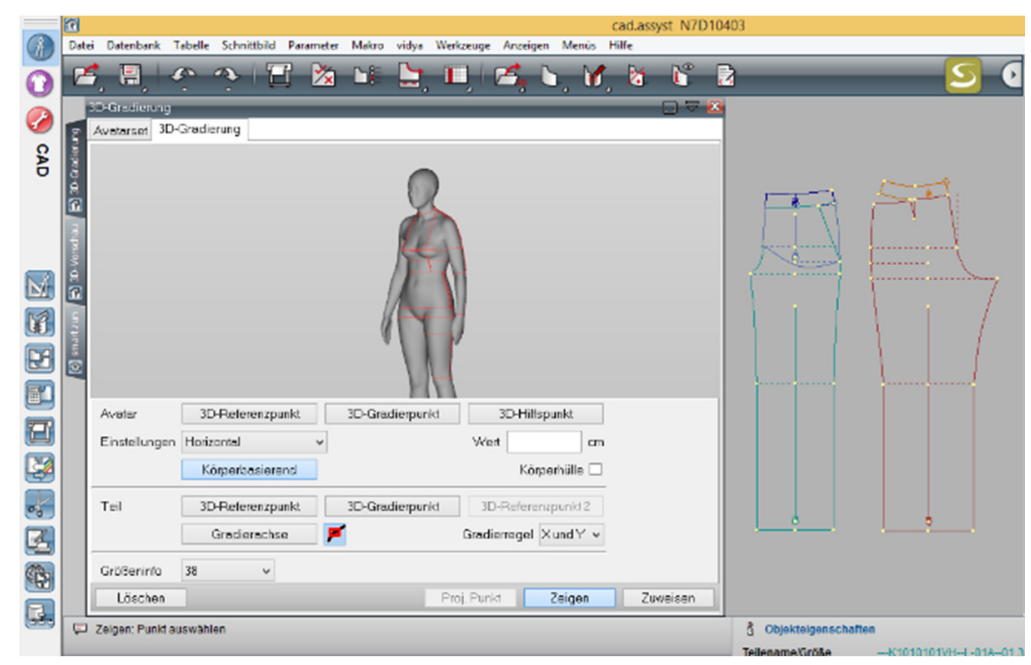

Fig. 6: 3D grading, assigned 3D grading to CAD pattern in vidyacad

\subsection{D Fit}

The generated scanatar files (obj. format) from selected subjects are exported and imported to 3D CAD systems available in VirLab (Virtual product development laboratory) at Niederrhein University: Vidya assyst, CLO3D Modelist, DC Suite, 3D Prototypig Lectra, OptiTex, Vstitcher Browzwear, Tuka3D.

All systems offer 3D fitting tests- slightly different- with virtual sewn garments and material characteristics. Different investigation tools are implemented by detecting body distance control, pressure and tension mapping, elasticity in warp, weft and bias direction, fit. 


\subsection{Products}

3D investigations are done taking basic products of skirts, dresses, trousers, blouses, shirts and jackets to check the fit of the modified pattern in an early state- before starting a manufacturing process. The pattern are prepared as interactive pattern (Grafis), macro pattern (smart developer/smart pattern assyst, Gerber wizard, Gemini mtm) or mtm graded pattern (Lectra, assyst, Gerber).

\section{Results}

The following results are only few extracts from the tests done with numerous females and males and the resulting fittings. They are divided up into three sections: The validation of measurements obtained from 3D full body scanning technology based on different scanning equipment, approach of implementing posture and shape parameters into $\mathrm{mtm}$ pattern construction and 3D Fit investigations for an accelerated pre-check of fit within the $\mathrm{mtm}$ process.

\subsection{D Full Body Scanning}

Fig. 7 shows the scanning results and extracted measurements of female F11 in scanning position standard and heelless, taken with different scanning technologies and evaluated in Anthroscan software compared to manual control.

Although the scanning technology is quite different in the process itself- apart from investment costs, handling and movability of the scanning equipment- if main task is reduced to getting data for a mass customization process the measurements vary only in a small window size and are acceptableespecially when compared to manually taken measurements.

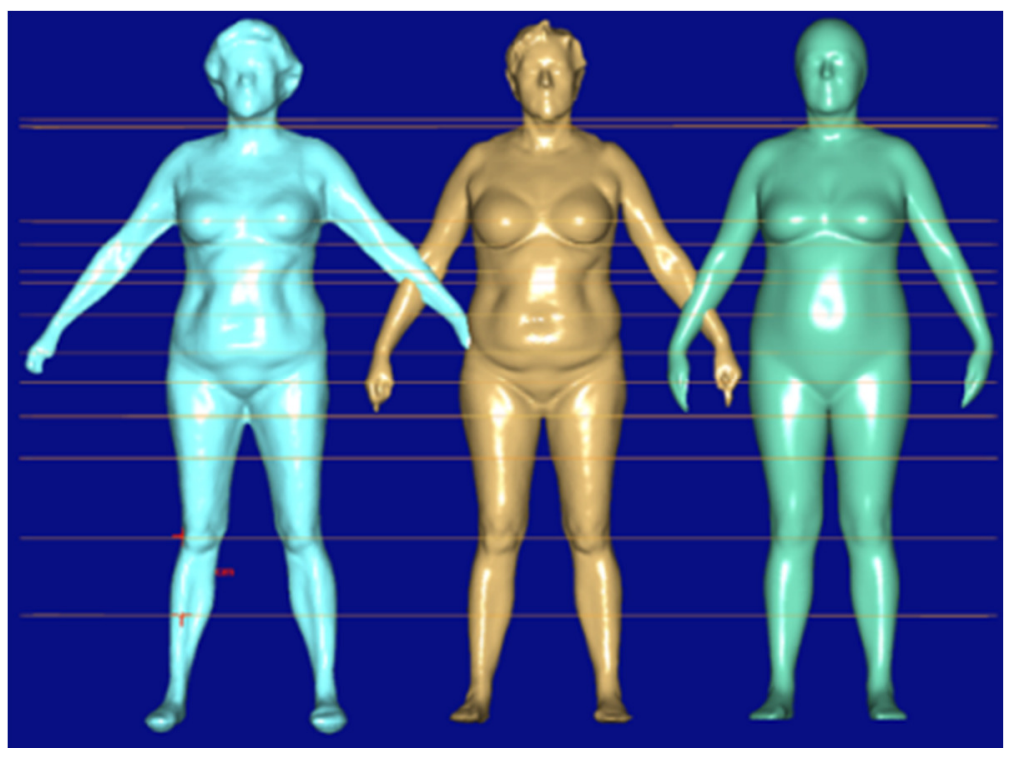

$\mathrm{K} 1-\mathrm{L}-\mathrm{K} 12$

\begin{tabular}{|l|l|l|l|l|}
\hline Measurement & \multicolumn{4}{|c|}{ Body Measurements [cm] } \\
\hline Technology & \multicolumn{1}{|c|}{ Styku (K1) } & \multicolumn{1}{|c|}{ Anthroscan (L) } & \multicolumn{1}{c|}{ Ditus (K12) } & Manually (M) \\
\hline body height & 167,8 & 166,4 & 167,6 & 166,9 \\
\hline neck 1 & 36,6 & 36,9 & 37,4 & 36,5 \\
\hline chest & 111,2 & 113,2 & 111,3 & 112,0 \\
\hline underbust & 76,9 & 76,4 & 77,8 & 76,0 \\
\hline waist 1 & 94,5 & 94,2 & 96,4 & 95,0 \\
\hline hip & 105,8 & 106,0 & 105,9 & 106,5 \\
\hline
\end{tabular}

Fig. 7: Extract of scan validation in Anthroscan and comparison of body measurements from different scanning systems with fixed plane locations; scanned subject $F_{11}$ 
Investigations of the scans from the sample size group show the most critical part: Getting the measurements on the right location- means the exact position where needed- for the pattern automatically. Fig. 8 shows the location problem in an overview of subject $M_{6}$ : also the different scanning systems are placing the measurement tapes differently and therefore a validation of the scan data by checking and if necessary replacing is compulsory necessary.
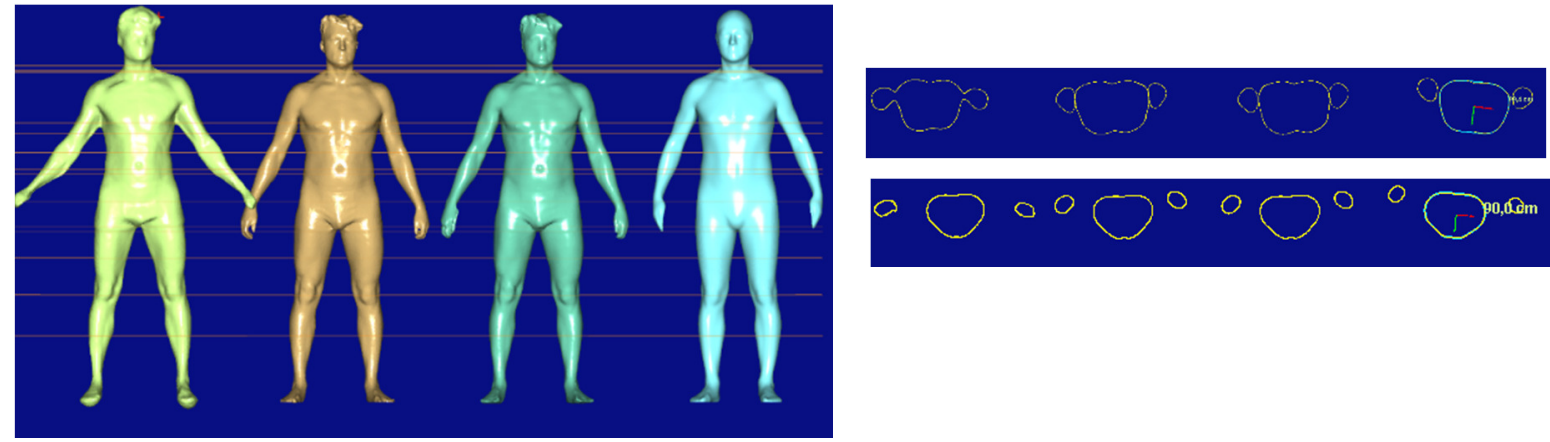

Fig. 8: Extract of scan investigation in Anthroscan- avatars generated from different scanning systems; location readjustment and plane section differences; scanned subject $M_{6}$

Fig. 9 shows the location problem in detail: The waist measurement location is one of the most important one- influencing most length measurements of the individual body system. By readjusting the position the waist girth value changes from $68,2 \mathrm{~cm}$ to $66,0 \mathrm{~cm}$ - this is already in mass production half of the grading value of the waist and therefore not acceptable at all for a made to measure process.

Also the product itself- design and position on body must be directly linked to the positioning of the virtual measurement tape.
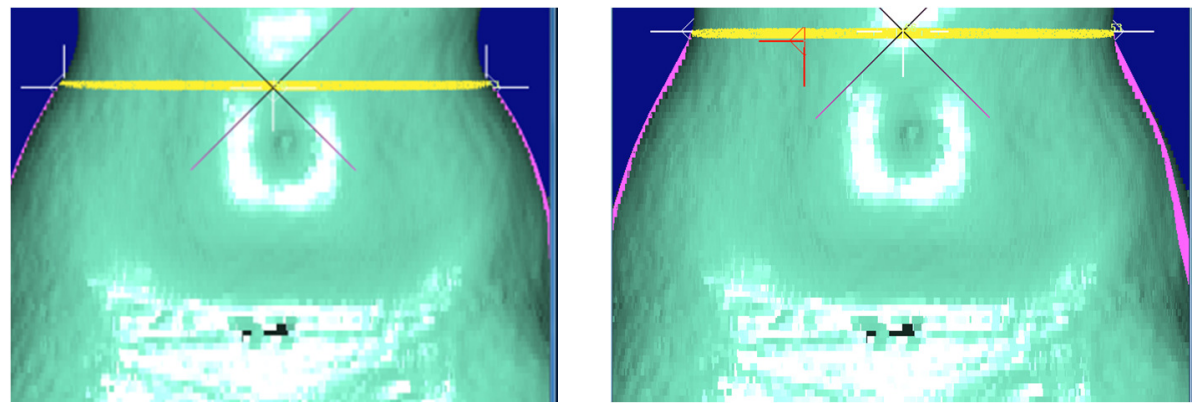

Fig. 9: Extract of scan investigation; waist readjustment according to body and product, scanned subject $F_{9}$

\subsection{Body posture and shape}

Apart from body measurements body posture and especially body shape have been investigated for checking the possibility to transfer body scanning information to CAD mtm pattern.

Regarding body shape the following areas contain important information worth to record and transfer to pattern product related:

- Hip curve HI/r, side seam Ssl/r (trousers, skirts, dresses, jackets)

- Shoulder slope Shr/l f/b (shirts, blouses, dresses, jackets)

- Armhole ahl $/ \mathrm{r}$ (shirts, blouses, dresses, jackets)

- Center back Bc1/2 (shirts, blouses, dresses, jackets)

- Bust dart Bdl/r St (shirts, blouses, dresses, jackets) 

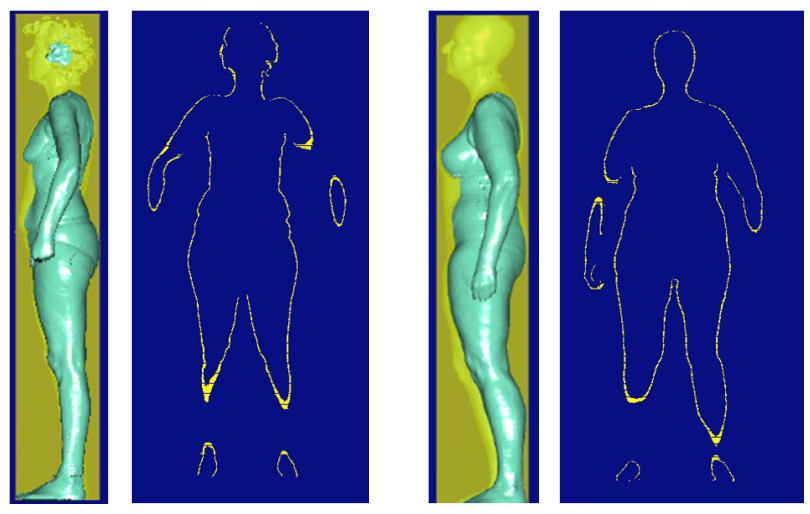

Fig. 10: Preparation of body shape contour extraction for CAD transfer, scanned subject $F_{2}$

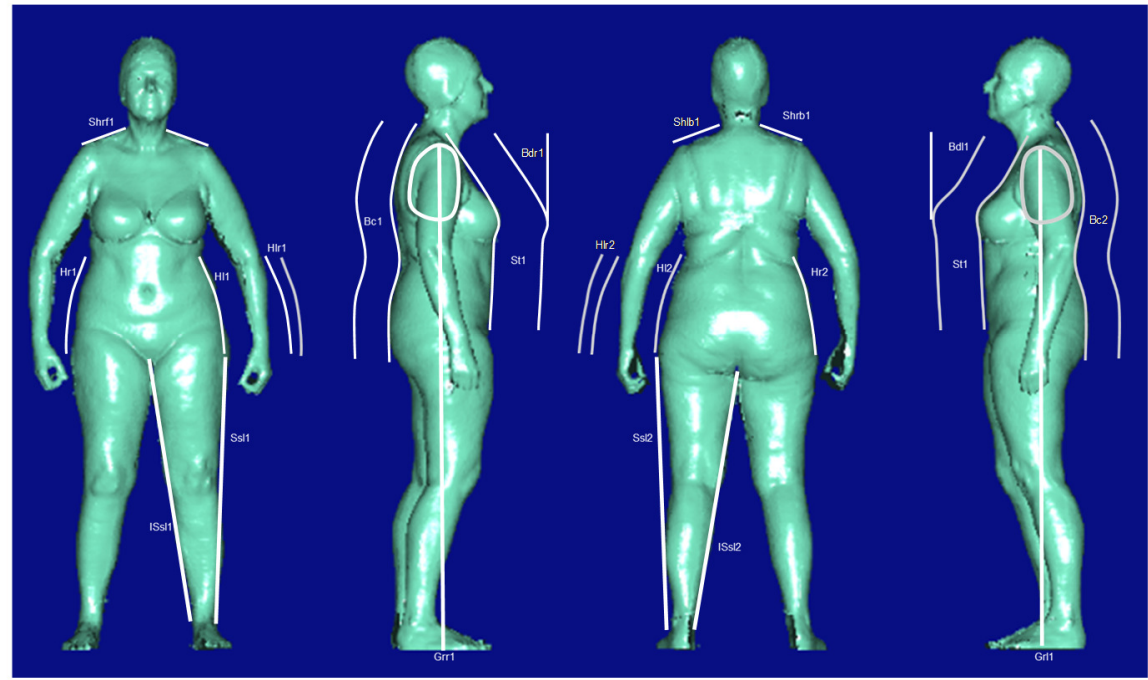

Fig. 11: Body shape contour extraction for $C A D$ export; scanned subject $F_{4}$

In fig. 12 the body shape lines are transferred to CAD pattern-basic block and model pattern. Due to the fact of limited shapes it is also possible to prepare so called "french curves" which can be assigned directly by activating or comparing and modulating automatically the body contour line with the database.
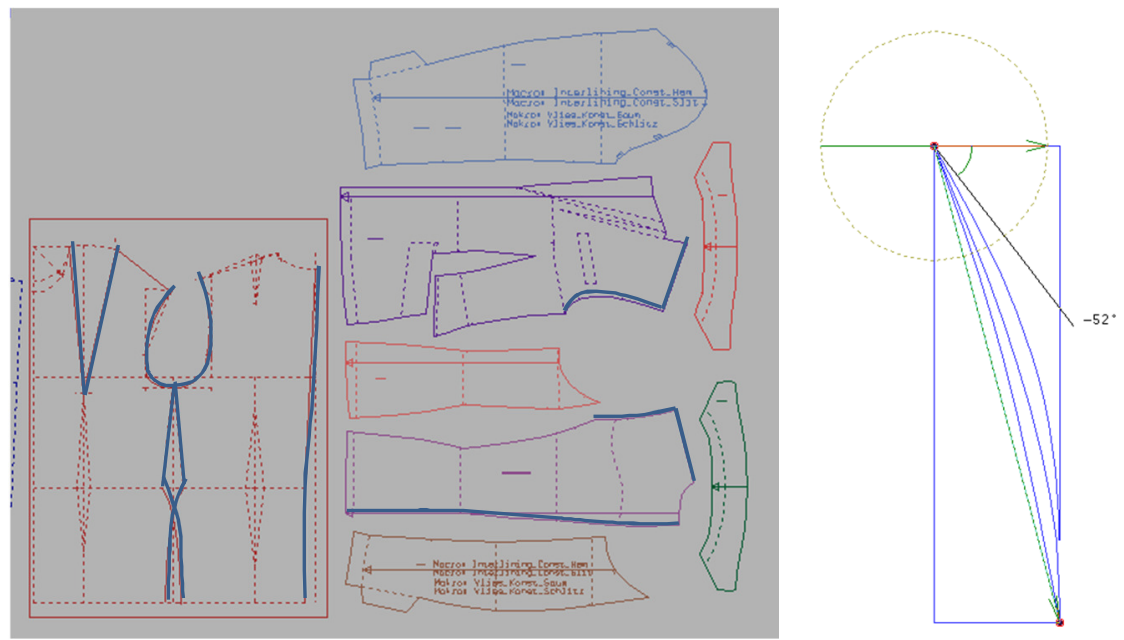

Fig. 12: Body shape contours transferred to CAD pattern in vidyacad, hip curve modulation 
Moving the 2D pattern directly on loaded background bodyscans offers the possibility to adapt the pattern interactively e.g. in Grafis system. Fig. 13 shows block modification cycles of $F_{12}$ to adapt the pattern to the body shape contour.
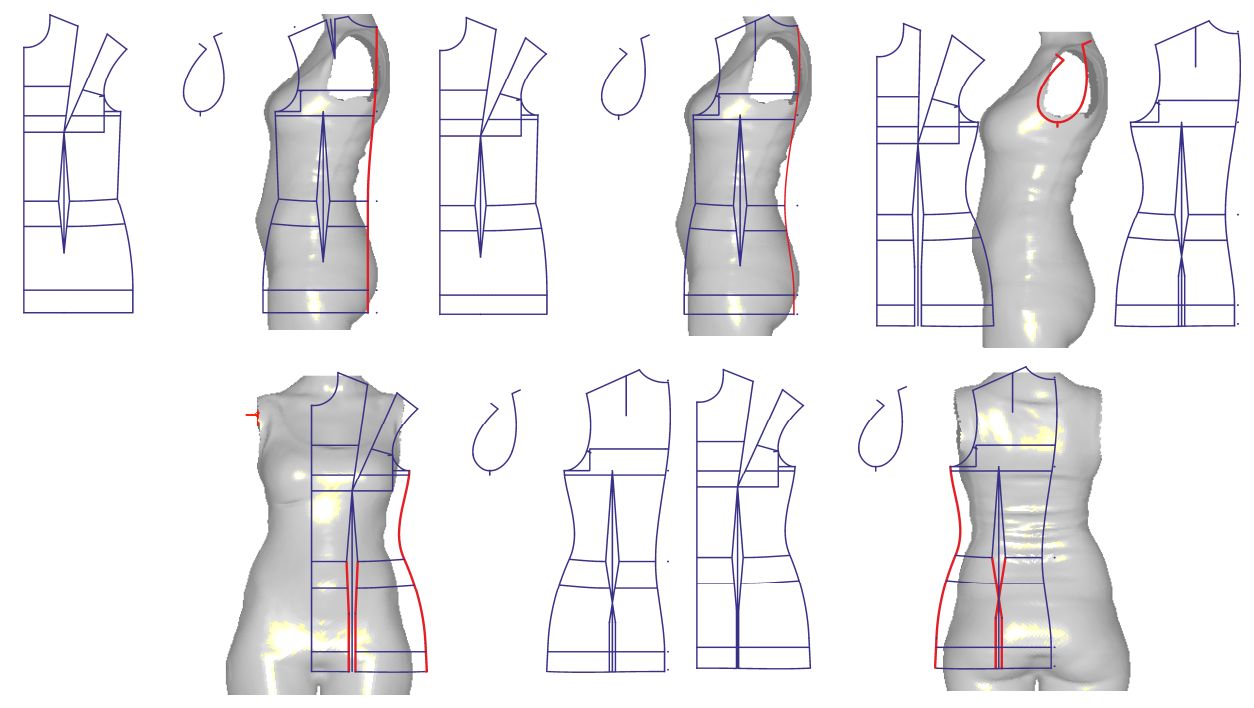

Fig. 13: Body shape contour adaption cycles, scanned subject $F_{12}$

For trousers products the most important fit region is front and back raise which comes together with the crotch curve running from center front waist to center back waist with an individual shape- usually responsible for a poor fit of the product. In Fig. 14 the crotch curve was also transferred from a prepared body scan to front and back panel of trousers, splitting both at the crotch depth point.

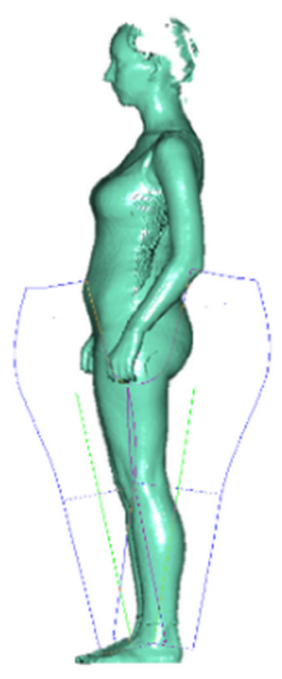

Fig. 14: crotch curve shape for female $F_{9}$

\subsection{D fit check}

Before starting manufacturing $\mathrm{mtm}$ clothing a virtual fitting is tested- not only for fit but also for a general understanding of shapes and styles. Therefore also a comparison was carried out to compare an individual $\mathrm{mtm}$ avatar- so called scanatar- and a statistical generated avatar, representing the morphological body type of the individual. For the measurements the body was analyzed in detail by re-measuring the most important ones accordingly to the construction system Contec (Niederrhein University) used for pattern construction. 
In Fig. 15 scanning subject $F_{1}$ was scanned in detail and measurements have been readjusted with regard to body and product. The information (measurements, morphological body contour) have been transferred to 2D CAD pattern. The fabric parameters (thickness, elasticity) have been also necessarily implemented. The product of a dress was virtually sewn and prepared in Vstitcher V7 to check fit. Modifications have been investigated when slightly changing morpho-parameters on one side and design aspects (design/style line position) screened.

When exchanging the real scanatar of the individual person and taking a statistical avatar representing the target mtm group it is obviously detected, that the fit- especially in the bust area changes. Thus statistical avatars- in the given procedure- are only limited useful in a $\mathrm{mtm}$ process or the parameters when creating these statistical avatars should be -from beginning on- more related to the morphological properties of the scanned subject. Therefore it is necessary to offer a practical solution for analyzing the data pool in detail and generating $\mathrm{mtm}$ body cluster types, individuals can be covered with. In fact a mtm solution does not have to offer a real tailor made solution, where all the details are implemented into the pattern and the product.
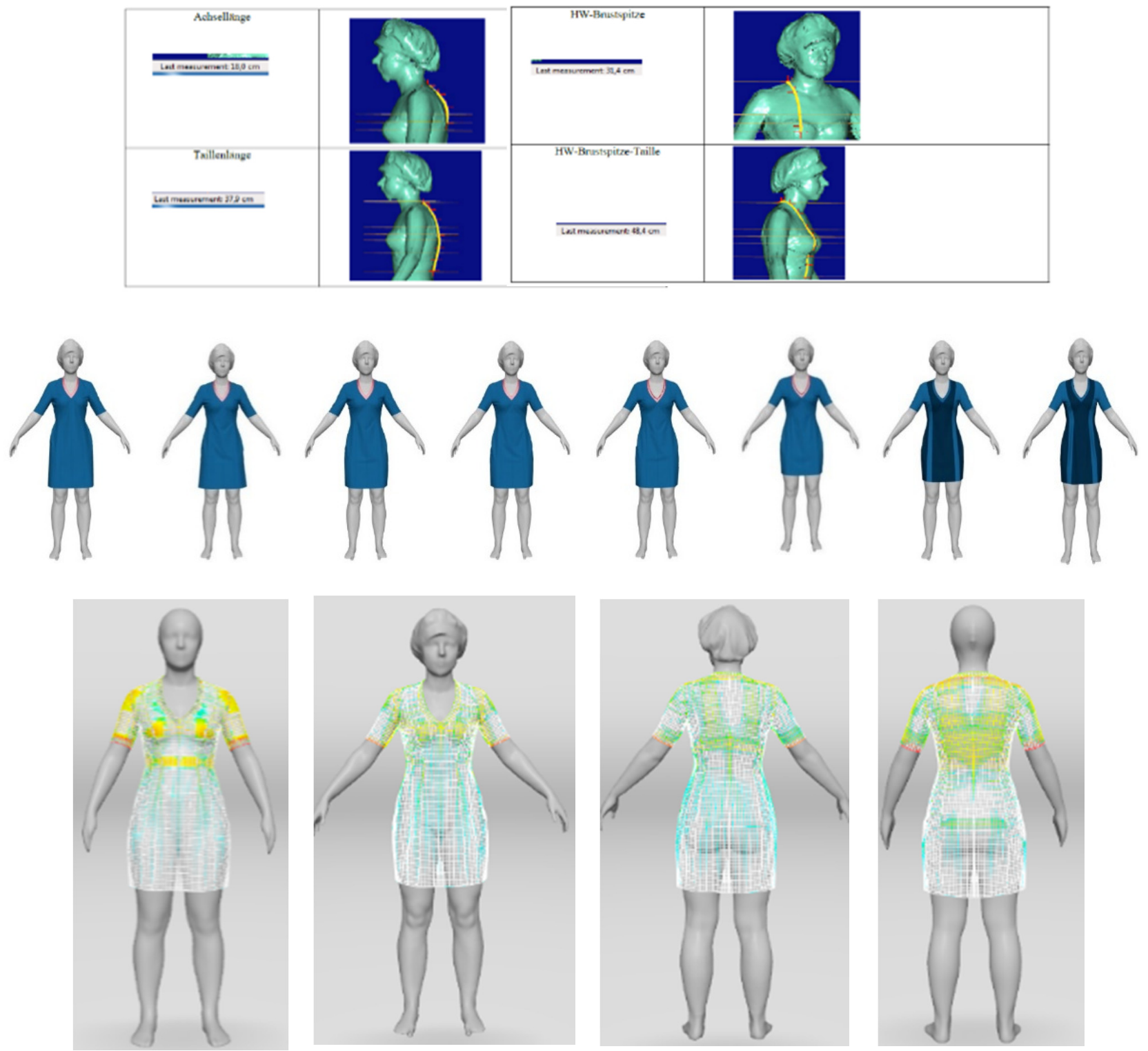

Fig. 15: scan validation, virtual fit and shaping, statistical avatar versus scanatar; scanned subject F8; material: knit-pre-tested on virtual drapometer of VirLab 


\section{Discussion and Conclusion}

The results of this paper have proven the importance and the benefit of transferring a mix of information to $\mathrm{mtm}$ pattern for getting best results. 3D full body scanning technology is a helpful tool to get these information quickly and available for longtime. Knowing that not all parameters can be fully controlled and few of them are changing hourly, most of them can be fixed and measurement extraction and scanatars are delivering valuable data. Therefore posture and position have a big impact on the results but can be controlled during the scanning process and offer additional information. Physical and mental impacts are hardly to control but facilitates on the other side a realistic view [1].

The algorithm to detect predefined measurements at best location on human bodies automatically and with high precision and quality is still not sufficient and research with focus on special measurements for garment is necessary to overcome these barriers. Up to now the scans have to be validated against the measurement locations which are predefined before transferring these measurements via $\mathrm{mtm}$ server to 2D CAD. Additionally body contour shapes can be transferred as well- generating construction elements or modifying given outer contour lines of pattern in shape, length and dart opening to adapt them to reality.

The $\mathrm{mtm}$ fit can be checked on scantars on one side and on statistical avatars generated from a database on the other side. The results show that the quality of an individual body scan is still delivering better results in testing fit. The statistical avatars are up to now the mean of created $\mathrm{mtm}$ clusters and therefore strongly dependent on the quality of how they are defined.

\section{References}

[1] M. Ernst and U. Detering-Koll.: "Posture Dependency of 3D-Body Scanning Data for a Virtual Product Development Process", in Proc. of 5th International Conference on 3D Body Scanning Technologies, Lugano, Switzerland, 21-22 October 2014, doi:10.15221/14.248

[2] H. Löffler-Wirth: "Body Typing of the adult population of Leipzig"; Application forum Human Solutions. Kaiserslautern, Germany, 21 April 2016

[3] S. Ashdown: "Sizing in Clothing", Woodhead Publishing, April 2007.

[4] M. Ernst and U. Detering-Koll: "Investigation on Body shaping Structures Using Body Scanning Technology and 3D Simulation Tools", in Proc. of $3^{\text {nd }}$ International Conference on 3D Body Scanning Technologies, Lugano, Switzerland, 16-17 October 2012, doi:10.15221/12.064

[5] M. Ernst: Next Generation of Product Development- Obtaining 3D Buy-in, Building Capability and Generating a Fruitful Roadmap; PI Conference Berlin, Germany, 5-6 October 2016

[6] F. Cordier, H. Seo, N. Magnenat-Thalmann: Made to Measure Technologies for an Online Clothing Store, IEE Computer Graphics and Applications; January/February 2003

[7] T. Spahiu, J. Kacani: 3D Body Scanning Technique for Anthropometric Measurements and Custom Clothing Designing, Conference Developing Third Mission Activities in Universities, International Conference, At Durres, Albania, September 2014 